\title{
Learning of Skilled Movements via Imitation in ASD
}

Danielle McAuliffe ${ }^{\mathrm{a}}$, Yi Zhao ${ }^{\mathrm{b}}$, Ajay S. Pillai ${ }^{\mathrm{a}, \mathrm{c}}$, Katarina Ament ${ }^{\mathrm{d}}$, Jack Adamek ${ }^{\mathrm{a}}$, Brian S. Caffo $^{\text {b }}$, Stewart H. Mostofsky ${ }^{\mathrm{d}, \mathrm{c}, \mathrm{e}}$, Joshua B. Ewen ${ }^{\mathrm{a}, \mathrm{c}, \mathrm{f}}$

aDepartment of Neurology and Developmental Medicine, Kennedy Krieger Institute, $707 \mathrm{~N}$ Broadway, Baltimore, MD 21205, USA

${ }^{b}$ Department of Biostatistics, Johns Hopkins Bloomberg School of Public Health, 615 N Wolfe St, Baltimore, MD 21205, USA

${ }^{\mathrm{c}}$ Department of Neurology, Johns Hopkins University School of Medicine, 855 N Wolfe St, Baltimore, MD 21205, USA

${ }^{\mathrm{d} C e n t e r}$ for Neurodevelopmental and Imaging Research, Kennedy Krieger Institute, $707 \mathrm{~N}$ Broadway, Baltimore, MD 21205, USA

e Department of Psychiatry and Behavioral Sciences, Johns Hopkins University School of Medicine, 600 N Wolfe St, Baltimore, MD 21287, USA

fDepartment of Psychological and Brain Sciences, Johns Hopkins University, 3400 N Charles St, Baltimore, MD 21218, USA

\section{Corresponding Author}

Joshua B. Ewen, MD

ewen@kennedykrieger.org

$+1-443-923-9170$

$+1-443-923-9540$ (fax)

RUNNING TITLE: Learning by Imitation in ASD

Grant Sponsor: NINDS/NIH; Grant numbers: R21 NS091569 (to JBE), R01 MH113652 (to JBE)

Grant Sponsor: NINDS/NIH; Grant number: R01 NS048527 (to SHM)

Number of Text Pages: 6 (excluding abstracts and bibliography)

Word Count: 3,568

Number of Figures: 4; Number of Tables: 1

This is the author's manuscript of the article published in final edited form as:

McAuliffe, D., Zhao, Y., Pillai, A. S., Ament, K., Adamek, J., Caffo, B. S., Mostofsky, S. H., \& Ewen, J. B. (2020). Learning of skilled movements via imitation in ASD. Autism Research, 13(5), 777-784. https://doi.org/10.1002/aur.2253 


\section{SCIENTIFIC ABSTRACT}

Autism spectrum disorder (ASD) consists of altered performance of a range of skills, including social/communicative and motor skills. It is unclear whether this altered performance results from atypical acquisition or learning of the skills or from atypical "online" performance of the skills. Atypicalities of skilled actions that require both motor and cognitive resources, such as abnormal gesturing, are highly prevalent in ASD and are easier to study in a laboratory context than are social/communicative skills. Imitation has long been known to be impaired in ASD; because learning via imitation is a prime method by which humans acquire skills, we tested the hypothesis that children with ASD show alterations in learning novel gestures via imitation. 18 participants with ASD and IQ>80, ages 8-12.9 years, and 19 typically developing (TD) peers performed a task in which they watched a video of a model performing a novel, meaningless arm/hand gesture and copied the gesture. Each gesture video/copy sequence was repeated 4-6 times. Eight gestures were analyzed. Examination of learning trajectories revealed that while children with ASD made nearly as much progress in learning from repetition 1 to repetition 4, the shape of the learning curves differed. Causal modeling demonstrated the shape of the learning curve influenced both the performance of overlearned gestures and autism severity, suggesting that it is in index of learning mechanisms relevant both to motor skills and autism core features. 


\section{LAY ABSTRACT}

Imitation is a route by which humans learn a wide range of skills, naturally and in therapies. Imitation is known to be altered in autism spectrum disorder (ASD), but learning via imitation has not been rigorously examined. We found that the shape of the learning curve is altered in ASD, in a way that has a significant impact both on measures of autism severity and of other motor skills.

Keywords: Dyspraxia, autism, imitation, learning, skill learning, motor control 
Children with autism spectrum disorder (ASD) demonstrate altered performance in a wide range of skills — social, language and motor. To date, it has been a challenge to find a single underlying deficit which is capable of explaining this broad range of impairments in seemingly unrelated domains of performance. Accounts from an information processing model may stress resources that are shared in the performance of each of these skills, whereas developmental psychological frameworks may examine how representations of skills are built up over time, through interactions with the environment. Happé (2001) took a significant step in making explicit this dichotomy by contrasting "online" (non-experience-dependent) and “developmental" (experience-dependent) performance deficits in ASD. The literature on procedural learning in ASD is somewhat limited when it comes to direct interrogation of complex skills (J.B. Ewen \& Mostofsky, 2012).

Imitation is one process by which skills are learned from interactions with the environment (Hoppitt \& Laland, 2013; Locke, 1693/1996; Meltzoff, Kuhl, Movellan, \& Sejnowski, 2009). Impaired imitative performance has been documented in ASD at least since the 1970s (DeMeyer et al., 1972; Edwards, 2014; Sevlever \& Gillis, 2010). Some theorists believe impaired imitation to be the central deficit in ASD (Rogers \& Pennington, 1991; Vivanti \& Rogers, 2014). Building on those theories, we have previously posited that impairments in learning via imitation contribute to autism-associated impairments in development not only of motor skills but of social/communicative skills as well (Mostofsky \& Ewen, 2011). There is evidence to support this notion, with impairments in imitation correlating with (Abrahams \& Geschwind, 2010; Sevlever \& Gillis, 2010; Williams, Whiten, \& Singh, 2004) and predicting (Toth, Munson, Meltzoff, \& Dawson, 2006) core symptom severity in ASD. Imitation-targeting interventions have a positive 
impact against ASD core symptoms (Ingersoll, 2010; Ingersoll \& Lalonde, 2010; Landa, Holman, O'Neill, \& Stuart, 2011).

While most social/communicative skills are difficult to control experimentally, motor skills serve as a more tractable model for the study of skill development. Imitation tasks bridge the divide by being social and communitive in nature but being scored via quantifiable motor behavior. Motor deficits were featured in Kanner's earliest description of autism (Kanner, 1943). Considerable work since that time has substantiated the high prevalence of motor deficits in individuals with ASD (Vanvuchelen, Roeyers, \& De Weerdt, 2011; Weimer, Schatz, Lincoln, Ballantyne, \& Trauner, 2001). In particular, ideomotor praxis (learned, skilled gestures for communicative or tool-use purposes) (Wheaton \& Hallett, 2007) has been shown multiple times to be widely impaired in ASD (Dewey, Cantell, \& Crawford, 2007; Dowell, Mahone, \& Mostofsky, 2009; Dziuk et al., 2007; MacNeil \& Mostofsky, 2012). Correlations between degree of impairment in motor skills and in core social/communicative diagnostic symptoms offer face validity for this claim, whether that mechanism is developmental in nature or an "online" capacity limitation (Dziuk et al., 2007).

The current work uses a paradigm in which we record changes in performance over the course of repeated imitation of a complex, novel, meaningless gesture. The development of skilled functional and communicative actions (contained within the concept of ideomotor praxis) are the focus of study, yet there are challenges in studying these actions directly. First, it is likely that many actions within the rubric of praxis would have already been learned to asymptotic levels, so a few additional trials in the laboratory would be underpowered to distinguish incremental learning. Second, it is plausible that participants in the ASD group may have, on average, 
different degrees of exposure/practice to communicative and tool-use gestures prior to the testing session, confounding the results.

The use of novel, meaningless gestures allows us to examine the imitative motoric output under the condition where all participants have no prior exposure to the specific gestures. Meaningless and meaningful/functional gestures have long been studied in parallel. Children with ASD show impairments in both gesture types (Mostofsky et al., 2006). Further, individuals with acquired apraxia often show impairments in production of both meaningful and meaningless gestures (for a nuanced discussion, see (Goldenberg, 2013), and chapter 6 in particular). The claim that related brain mechanisms subserve the production of both types of gestures is substantiated both by lesion (Goldenberg, 2013) and physiological imaging data (J. B. Ewen et al., 2016).

The overall goal of the work was to evaluate the hypothesis that deficits in praxis performance could be due to altered learning of those gestures via imitation, and that the underlying alteration of learning mechanisms also underlies the presence and severity of ASD symptoms.

To test these hypotheses, we first examined rates of learning (improving performance) over the course of repeated imitation of a number of individual gestures. We next used statistical approaches to evaluate causal models that specify the influence of learning curve trajectory (as calculated from the novel gesture imitation task) on autism diagnosis or severity and on praxis performance (as derived from a clinical praxis measure). Because ASD severity is known to correlate with praxis performance (Dziuk et al., 2007), the models also include a path from diagnostic symptoms to praxis ability. Finally, the models control for the influence that motor coordination could have on the measurement of praxis performance. 


\section{Materials and Methods}

\section{Participants}

Both ASD and typically developing control (TD) groups included children aged 8.0-12.9 years. Participants in the ASD group met criteria for ASD based on the clinical judgment of an experienced ASD clinician and researcher (SHM), as confirmed using the Autism Diagnostic Observation Schedule-2, Module 3 (ADOS-2 (Lord et al., 2000)); and, for 12 of 18 in the ASD group, the Autism Diagnostic Interview-Revised (ADI-R; (Lord, Rutter, \& Le Couteur, 1994)), all of whom met criteria.

Exclusion criteria for both groups included full-scale IQ $<80$ on the Wechsler Intelligence Scale for Children - IV (D Wechsler, 2003) or - V (D. Wechsler, 2014), history of a definite neurological disorder (e.g., seizures, tumors, traumatic brain injury, stroke) and presence of a severe chronic medical disorder, visual impairment, history of substance abuse or dependence, or presence of childhood schizophrenia or psychosis. Additional exclusion for the ASD group included history of known etiology for autism (e.g., tuberous sclerosis) and history of documented prenatal/perinatal insult. We excluded children with ASD if they met criteria for any diagnosis other than attention-deficit/hyperactivity disorder or anxiety disorders on the Kiddie Schedule for Affective Disorders and Schizophrenia for School-Aged Children (K-SADS) (Kaufman et al., 2013); given the high comorbidity of these disorders with ASD, it was not practical to exclude these co-occurring diagnoses. Additional exclusion for the TD group included Social Responsiveness Scale (SRS) (Constantino \& Gruber, 2005) scores outside the normal range, history of a developmental disorder or a psychiatric disorder (evaluated via the KSADS) and having an immediate family member with an ASD diagnosis. 
All participants were right-handed, based on the Edinburgh Handedness Inventory results of 0.5 to 1 (Oldfield, 1971).

Three participants (2 ASD group and 1 TD) were excluded from analyses because scoring of their performance failed to reach the inter-rater reliability threshold of $70 \%$. Analyses included data from 18 participants with ASD and 19 TD controls (Table 1). All participants received the WISC-V, except for two with ASD and one control who received the WISC-IV. Group comparisons were made among participants who received the WISC-V. Children with ASD had ADOS total scores between 7 and 20. Within the ASD group, some participants met criteria on the K-SADS for Attention-Deficit/Hyperactivity Disorder (10 children), Specific Phobia (2), Generalized Anxiety Disorder (3) and Oppositional Defiant Disorder (1), Social Anxiety Disorder (2). No TD participant met criteria for any diagnosis on the K-SADS. Five participants with ASD were on chronic medications: one was on dextroamphetamine and risperidone, one was on clonidine, two were on methylphenidate, and one was on montelukast and levocetirizine.

The protocol was approved by Johns Hopkins Medicine Institutional Review Board. Written consent was obtained from parents and, when possible, from participants. Otherwise, verbal assent was obtained from participants.

\section{Procedures}

Children in both groups participated in several research studies over two days of testing, all with similar inclusion/exclusion criteria. Schedules were variable based on which combination of studies an individual participant engaged in and tester availability. IQ testing was performed early on the first day for both groups, and ADOS was performed in the morning of the first day for participants in the ASD group. Additional tests relevant to the current work included the 
Physical and Neurological Assessment of Subtle Signs (PANESS) (Denckla, 1985) which examines for movement speed, coordination and precision. The PANESS total score was used as an index of coordination in the causal models. The pediatric adaptation of the Florida Apraxia Battery (FAB) (Mostofsky et al., 2006) consists of single-trial imitation of novel gestures as well as the performance of age-appropriate transitive and intransitive gestures to command (e.g., "Show me how you wave 'bye."), and transitive gestures via the use of a provided toy (e.g., comb). FAB, PANESS and the experimental task were performed variably over the first or second research days.

\section{Task}

This study employed a novel-gesture-learning task previously studied in neuro-typical adults (J. B. Ewen et al., 2016). In each trial (Fig. 1), the participant watched a video of the right arm of a model performing a novel, meaningless gesture. The whole model was not shown in order to minimize potential confounds from group differences in the "audience effect" (Chevallier et al., 2014) and in visual attention (Chevallier et al., 2014; Falck-Ytter, Fernell, Hedvall, von Hofsten, \& Gillberg, 2012). Videos were displayed on an Acer V196L 19" display, about 36" from the participants' eyes. Following a Go cue (fixation cross turning green), the participant then performed the gesture. Feedback was given by a research assistant (trained and research-reliable in the coding of the pediatric modification of the Florida Apraxia Battery (Mostofsky et al., 2006)) as to whether the participant performed the gesture correctly or incorrectly. If the gesture was performed incorrectly, then the research assistant directed the participant to "Notice how [the model] moved her fingers," or similar feedback. The rationale behind the feedback was that children would be unlikely to improve unless given some feedback, but we restricted precise 
guidance so as not to make the movement information verbally mediated. The video was then repeated, and the participant again copied the movement. Video blocks contained three gestures in fixed order; block order was randomized across participants.

There was a total of 12 gestures. Fifty percent of gestures had a total of four repetitions; $25 \%$, five repetitions; and 25\%, six repetitions. Because of an anecdotal observation that children may have better performance on the early and last repetitions, we wanted the total number of repetitions for any one gesture to be somewhat unpredictable so as to motivate equal effort across all repetitions.

The movements were judged correct if all elements were performed, and in the correct order (see Supplementary Table S1). Gestures were not considered incorrect if the direction of rotation (e.g., on "C Twist") was different than the model's.

\section{Scoring}

Scoring on each trial (correct/incorrect) was conducted in real time by the research assistant who provided feedback. In addition, the scoring was repeated offline by a single rater who was blinded to the original (real-time) scoring results. Reliability was assessed on a participant-byparticipant, and gesture-by-gesture basis. All gestures $\times$ repetitions were scored for each participant. Any individual gesture that failed to reach $80 \%$ concurrence between raters was excluded from analysis. After eliminating gestures, any participant who had less than $75 \%$ agreement on all remaining gestures $\times$ repetitions would be removed from further analysis. The scoring from the real-time raters was used for subsequent analysis for all participants who passed reliability. 
Analysis

Participant-specific performance was scored at each repetition as fraction of gestures performed correctly. Statistical testing was restricted to the first four repetitions, since all gestures were repeated at least four times.

The analysis plan had two primary steps: the first to examine potential group differences in the learning curves, and the second to examine whether individual differences correlate with clinical and experimental results as specified by a causal model that relates learning rate, diagnosis/autism severity, motor coordination and overlearned praxis ability. The analysis of causal models is similar to structural equation modeling and allows for the assessment of the effect of factors on one another, while controlling for both quantified and unquantified confounds (J. Pearl, 2009).

Group differences in the learning curve (Fig. 2) were first examined using a mixed-effects model, with group (diagnosis), repetition and their interactions as the fixed terms, as well as age, sex and motor coordination, as measured by the PANESS total score, as well as a participantspecific random effect. We next conducted separate Markov models at repetitions 2, 3 and 4. Markov models, in this context, measure learning by regressing the participant's performance at the immediate prior repetition as well as the interaction with diagnosis, to study the disparity of learning rate between groups. We again adjusted for age, sex and PANESS.

We measured the association among the learning curve, praxis performance and core ASD symptoms by specifying and then testing two explicit causal models (J. Pearl, 2001): one involving both groups (Fig. 3a) and one examining data only from the ASD group (Fig. 3b). To quantify the shape of the learning curve within a single variable, we performed a principal 
components analysis (PCA) on number of gestures performed correctly at each of the 4

repetition, across participant (pooling the two groups). Our prime testable theoretical claims were (1) that rate of skill learning, as evidenced by the rate of improvement in the novel gesture imitation paradigm, impacts overlearned praxis skill, as evidenced on the pediatric adaptation of the FAB (causal arrow $\delta$, Figs. 3a, 3b), and (2) that rate of skill learning impacts core diagnostic features of ASD, as evidenced by presence/absence of ASD diagnosis in the first model and ADOS score in the second (causal arrow $\alpha$ ). Beyond these two causal claims under investigation, it is also known that children with ASD show worse performance on the PANESS and FAB, in a way that correlates with ADOS score (causal arrows $\beta$ and $\gamma_{1}$ ) (Mostofsky et al., 2006), and that PANESS predicts poorer FAB performance $\left(\gamma_{2}\right)$ (Dziuk et al., 2007).

\section{Results}

\section{Behavioral Results}

Four gestures were excluded from analysis based on having an inter-rater reliability of $<75 \%$. Across the four trials, children with ASD showed poorer imitation performance (Fig. 2), with significant diagnosis-related differences at each repetition in the mixed-effects model. Overall, TD children's performance improved by $4.47 \pm 0.50$ (mean $\pm \mathrm{SD} ; p<0.000)$ gestures out of 8 from repetition 1 to repetition 4 . The performance of children in the ASD group improved on average by 3.73 gestures, for a "very small" to "small" between-group effect size (Cohen's $d=0.17$; $p=0.33$ ) (Sawilowsky, 2009). With regard to the rate of improvement across repetitions, the Markov models revealed a marginal (uncertain) result in terms of group differences in the rate of learning between repetitions 1 and $2(p=0.087)$, a significant difference in learning between repetitions 2 and 3 ( $p=0.035)$, and no statistical difference between repetitions 3 and 4 ( $p=0.224)$. 
To summarize, the learning curves between groups diverge in the early trials, with marginally significant difference at repetition 2 and significant difference at repetition 3 , but re-converge by the final, repetition 4 .

To test whether these differences in the learning curve represent a theoretically significant difference with impact on core ASD symptoms (as measured by ASD diagnosis and by ADOS) and on praxis skill development (as measured by FAB), we examined the results of mediation analysis via our causal models. Due to missing data, we were able to analyze data from 13 participants with ASD and 20 TD peers. We first examined the loading of the first principal component (PC1) (Fig. 4) as a measure of shape of the learning curve. PC1 accounted for $86 \%$ of the variance and reflected a mean shift of the 4 repetitions. PC2 (10\% of variance) was used as a covariate in the models because it was heavily weighted on repetition 1 and adjusting for PC2 therefore allowed us to remove the effect of initial (non-learning) imitation ability. Given that PC2 was almost exclusively weighted in repetition 1, it appears to be reflective of learningindependent (baseline) imitation performance. PC1 (adjusting for PC2, as well as age and sex) was therefore used in the analysis of the causal model as reflective of the shape of the learning curve. Within the ASD-group-only model, the total effect of PC1 on FAB total correct score was positive, large, and significant (Cohen's $d=0.90 ; p=0.010$; higher FAB $=$ better performance). The effect of PC1 on ADOS was negative, moderate and significant (Cohen's $d=-0.67 ; p=0.038$; lower $\mathrm{ADOS}=$ less severe symptoms). PC1 (learning-dependent imitiative performance) explained about $66 \%$ of the variation in FAB, while PC2 (learning-independent imitative performance) explained about $1 \%$ of the variance.

Within the model considering both groups, the total effect of PC1 (adjusting for PC2, age and sex) on FAB total correct score was positive, large and significant (Cohen's $d=1.24 ; p<0.001$ ), 
with a significant path effect from learning-curve shape through diagnosis to FAB score (explains $31.1 \%$ of the total effect of $\mathrm{PC} 1$ [adjusted] on $\mathrm{FAB}$ ). The effect of $\mathrm{PC} 1$ on diagnosis was moderately significant (Cohen's $d=-0.68 ; p=0.001$ ), with higher PC1 score predictive of typical development.

Stated another way, the shape of the learning curve captures an effect that is relevant both for core ASD symptomatology and of previously acquired praxis skills.

\section{Discussion}

The primary aim of this work was to determine whether children with ASD show altered learning curves associated with practicing the imitation of novel gestures. Children with ASD showed subtle differences in the shape of their learning curves, compared with TD peers. Although they began to catch up in terms of novel gesture imitation performance gains by the end of the task, nevertheless the shape of their altered learning curves was significantly associated with the diagnosis of ASD and ASD severity (assessed using the ADOS), as well as their development of praxis competence.

Children in both groups improved their ability on average to imitate each gesture over the course of practice. The shapes of the curves, however, differed. TD children plateaued in their performance gains early, and children with ASD began to catch up by the fourth repetition. Despite apparent convergence in magnitude of learning, the shape of the learning curve nevertheless had a significant between-group and within-ASD impact on eventual performance of overlearned praxis as well as on social abilities within the ASD group.

These results provide some evidence supporting a developmental impact of poor imitative gestural learning on autism-associated impairments in social and motor skill development, and 
support our hypothesis that the motor dyspraxia typical of ASD and the "social dyspraxia" that is diagnostic of the disorder may be commonly related to altered skill-learning mechanisms (Mostofsky \& Ewen, 2011).

An overarching goal of this line of research is to parse the contribution to the ASD phenotype (altered social and motor skill performance) of "developmental" (experience-dependent) and "online" (non-experience-dependent) mechanisms. The group difference in performance at repetition 1 (which is, by definition, not experience dependent) demonstrates the presence of online differences in imitative function. Taken together with the differences in learning curve shape, there was evidence for both experience-dependent and -independent alterations in motor skill performance. The impact of the two mechanisms, however, was different in terms of the causal influence on praxis performance: the causal statistical model within the ASD-only group showed that learning curve shape explained about $66 \%$ of the variance in praxis performance as measured on the $\mathrm{FAB}$, whereas the learning-independent performance (approximating performance at repetition 1) accounted for about $1 \%$ of performance on the FAB.

These results need to be interpreted with some caution when attempting to understand how social skills or even praxis skills are acquired. Because skill learning that occurs on different timescales is known to depend on different neurobiological mechanisms (Kelly \& Garavan, 2005), it is possible that a single-session and highly controlled learning paradigm may not fully capture the effect of interest with regard to social skills, communicative skills and highly learned tool-use skills that are practiced naturalistically within dozens or hundreds of "trials," over the entire lifespan. More ecologically relevant future work should involve the training of motor and social skills over many months, with performance measured at multiple time points along the way. Of note, however, altered learning curves have also been described in ASD using a 
different form of skill learning from the one studied here: adaptation (Izawa et al., 2012).

Moreover, how the altered acquisition of these skills could affect developmental trajectories is a question that dates back as far as Vygotsky (Vygotskiī, 1978).

We also note that, while our measurement procedure was sufficiently powerful to uncover a group effect (and one which correlated with clinically important measures), even more subtle and specific differences could be uncovered using motion capture and application of computer vision methods (in contrast to human observer coding used here and in all prior studies). At least one diagnostic biomarker has been developed based on measurement of movement kinematics $(\mathrm{Wu}$, Jose, Nurnberger, \& Torres, 2018). One could imagine examining, in a data-driven fashion, the specific derived variables that separate praxis performance between the two groups, and then measuring those variables specifically within a learning paradigm.

In conclusion, children with ASD demonstrate mild difference in the rates at which they learn to imitate novel, meaningless gestures compared with peers. However, causal statistical analysis demonstrates an effect of the shape of the learning curve on measures of both praxis performance and ASD diagnosis/severity. 
Conflict of Interest: The authors have no relevant conflict of interests. 


\section{References}

Abrahams, B. S., \& Geschwind, D. H. (2010). Connecting genes to brain in the autism spectrum disorders. Arch Neurol, 67(4), 395-399. doi:67/4/395 [pii]

Chevallier, C., Parish-Morris, J., Tonge, N., Le, L., Miller, J., \& Schultz, R. T. (2014).

Susceptibility to the audience effect explains performance gap between children with and without autism in a theory of mind task. J Exp Psychol Gen, 143(3), 972-979. doi:10.1037/a0035483

Constantino, J., \& Gruber, C. (2005). Social Responsiveness Scale. Los Angeles, CA: Western Psychological Services.

DeMeyer, M. K., Alpern, G. D., Barton, S., DeMyer, W. E., Churchill, D. W., Hingtgen, J. N., , . . Kimberlin, C. (1972). Imitation in autistic, early schizophrenic, and non-psychotic subnormal children. J Autism Child Schizophr, 2(3), 264-287.

Denckla, M. B. (1985). Revised neurological examination for subtle signs. Psychopharmacology Bulletin, 21, 773-779.

Dewey, D., Cantell, M., \& Crawford, S. G. (2007). Motor and gestural performance in children with autism spectrum disorders, developmental coordination disorder, and/or attention deficit hyperactivity disorder. J Int Neuropsychol Soc, 13(2), 246-256. doi:S1355617707070270 [pii]

Dowell, L. R., Mahone, E. M., \& Mostofsky, S. H. (2009). Associations of postural knowledge and basic motor skill with dyspraxia in autism: implication for abnormalities in distributed connectivity and motor learning. Neuropsychology, 23(5), 563-570. doi:200912548-003 [pii]

Dziuk, M. A., Gidley Larson, J. C., Apostu, A., Mahone, E. M., Denckla, M. B., \& Mostofsky, S. H. (2007). Dyspraxia in autism: association with motor, social, and communicative deficits. Dev Med Child Neurol, 49(10), 734-739. doi:DMCN734 [pii]

Edwards, L. A. (2014). A meta-analysis of imitation abilities in individuals with autism spectrum disorders. Autism Res, 7(3), 363-380. doi:10.1002/aur.1379 
Ewen, J. B., \& Mostofsky, S. H. (2012). Procedural Memory. In F. R. Volkmar (Ed.), Encyclopedia of Autism Spectrum Disorders. New York: Springer.

Ewen, J. B., Pillai, A. S., McAuliffe, D., Lakshmanan, B. M., Ament, K., Hallett, M., . . . Mostofsky, S. H. (2016). Practicing Novel, Praxis-Like Movements: Physiological Effects of Repetition. Front Hum Neurosci, 10, 22. doi:10.3389/fnhum.2016.00022

Falck-Ytter, T., Fernell, E., Hedvall, A. L., von Hofsten, C., \& Gillberg, C. (2012). Gaze performance in children with autism spectrum disorder when observing communicative actions. J Autism Dev Disord, 42(10), 2236-2245. doi:10.1007/s10803-012-1471-6

Goldenberg, G. (2013). Apraxia: The cognitive side of motor control. Oxford, UK: Oxford University Pres.

Happe, F. (2001). Social and Nonsocial Development in Autism: Where are the Links? In The Development of Autism: Persepctives from Theory and Research (pp. 237-254): Routledge.

Hoppitt, W., \& Laland, K. (2013). Social Learning: An Introduction ot Mechanisms, Methods, and Models. Princeton: Princeton University Press.

Ingersoll, B. (2010). Pilot randomized controlled trial of Reciprocal Imitation Training for teaching elicited and spontaneous imitation to children with autism. J Autism Dev Disord, 40(9), 1154-1160. doi:10.1007/s10803-010-0966-2

Ingersoll, B., \& Lalonde, K. (2010). The impact of object and gesture imitation training on language use in children with autism spectrum disorder. J Speech Lang Hear Res, 53(4), 1040-1051. doi:10.1044/1092-4388(2009/09-0043)

Izawa, J., Pekny, S. E., Marko, M. K., Haswell, C. C., Shadmehr, R., \& Mostofsky, S. H. (2012). Motor learning relies on integrated sensory inputs in ADHD, but over-selectively on proprioception in autism spectrum conditions. Autism Res, 5(2), 124-136. doi:10.1002/aur.1222

Kanner, L. (1943). Autistic disturbances of affective conduct. Nervous Child, 2, 217-250.

Kaufman, J., Birmaher, B., Axelson, D., Perepletchikova, F., Brent, D., \& Ryan, N. (2013). Kiddie Schedule for Affective Disorders and Schizophrenia for School-Aged Children -- 
Lifetime Version (Kiddie-SADS-PL 2013 Working Draft). Pittsburgh, PA: Western Psychiatric Institute and Clinic and Yale University.

Kelly, A. M., \& Garavan, H. (2005). Human functional neuroimaging of brain changes associated with practice. Cereb Cortex, 15(8), 1089-1102. doi:bhi005 [pii]

Landa, R. J., Holman, K. C., O'Neill, A. H., \& Stuart, E. A. (2011). Intervention targeting development of socially synchronous engagement in toddlers with autism spectrum disorder: a randomized controlled trial. J Child Psychol Psychiatry, 52(1), 13-21. doi:10.1111/j.1469-7610.2010.02288.x

Locke, J. (1693/1996). Some Thoughts Concerning Education (R. W. Grant \& N. Tarcov Eds.). Indianapolis, IN: Hackett Publishing Company, Inc.

Lord, C., Risi, S., Lambrecht, L., Cook, E. H., Jr., Leventhal, B. L., DiLavore, P. C., . . Rutter, M. (2000). The autism diagnostic observation schedule-generic: a standard measure of social and communication deficits associated with the spectrum of autism. J Autism Dev Disord, 30(3), 205-223.

Lord, C., Rutter, M., \& Le Couteur, A. (1994). Autism Diagnostic Interview-revised: A revised version of a diagnostic interview for caregivers of individuals with possible pervasive developmental disorders. Journal of Autism and Developmental Disorders, 24, 659-685.

MacNeil, L. K., \& Mostofsky, S. H. (2012). Specificity of dyspraxia in children with autism. Neuropsychology, 26(2), 165-171. doi:2012-02234-001 [pii]

Meltzoff, A. N., Kuhl, P. K., Movellan, J., \& Sejnowski, T. J. (2009). Foundations for a new science of learning. Science, 325(5938), 284-288. doi:10.1126/science.1175626

Mostofsky, S. H., Dubey, P., Jerath, V. K., Jansiewicz, E. M., Goldberg, M. C., \& Denckla, M. B. (2006). Developmental dyspraxia is not limited to imitation in children with autism spectrum disorders. J Int Neuropsychol Soc, 12(3), 314-326.

Mostofsky, S. H., \& Ewen, J. B. (2011). Altered connectivity and action model formation in autism is autism. Neuroscientist, 17(4), 437-448. doi:1073858410392381 [pii]

Oldfield, R. (1971). The assessment and analysis of handedness: The Edinburgh Inventory. Neuropsychologia, 9, 97-113. 
Pearl, J. (2001). Direct and Indirect Effects. Paper presented at the Proceedings of the Seventeenth Conference on Uncertainty in Artificial Intelligence, San Francisco, CA. Pearl, J. (2009). Causality (second ed.). Cambridge: Cambridge University Press.

Rogers, S. J., \& Pennington, B. F. (1991). A theoretical approach to the deficits in infantile autism. Development and Psychopathology, 3(2), 137-162.

Sawilowsky, S. S. (2009). New Effect Size Rules of Thumb. Journal of Modern Applied Statistical Methods, 8(9), Article 26. doi:Journal of Modern Applied Statistical Methods

Sevlever, M., \& Gillis, J. M. (2010). An examination of the state of imitation research in children with autism: Issues of definition and methodology. Res Dev Disabil, 31(5), 976-984. doi:S0891-4222(10)00093-4 [pii]

Toth, K., Munson, J., Meltzoff, A. N., \& Dawson, G. (2006). Early predictors of communication development in young children with autism spectrum disorder: joint attention, imitation, and toy play. J Autism Dev Disord, 36(8), 993-1005. doi:10.1007/s10803-006-0137-7

Vanvuchelen, M., Roeyers, H., \& De Weerdt, W. (2011). Imitation assessment and its utility to the diagnosis of autism: evidence from consecutive clinical preschool referrals for suspected autism. J Autism Dev Disord, 41(4), 484-496. doi:10.1007/s10803-010-1074-z

Vivanti, G., \& Rogers, S. J. (2014). Autism and the mirror neuron system: insights from learning and teaching. Philos Trans R Soc Lond B Biol Sci, 369(1644), 20130184.

doi:10.1098/rstb.2013.0184

Vygotskiǐ, L. S. (1978). Interaction between Learning and Development. In M. Cole (Ed.), Mind in society: The development of higher psychological processes (pp. 79-91). Cambridge: Harvary University Press.

Wechsler, D. (2003). Wechsler intelligence scale for children, fourth edition. . San Antonio, TX: The Psychological Corporation.

Wechsler, D. (2014). Wechsler intelligence scale for children-fifth edition. Bloomington, MN: PsychCorp. 
Weimer, A. K., Schatz, A. M., Lincoln, A., Ballantyne, A. O., \& Trauner, D. A. (2001). "Motor" impairment in Asperger syndrome: evidence for a deficit in proprioception. J Dev Behav Pediatr, 22(2), 92-101. Retrieved from https://www.ncbi.nlm.nih.gov/pubmed/11332785

Wheaton, L. A., \& Hallett, M. (2007). Ideomotor apraxia: a review. J Neurol Sci, 260(1-2), 1-10. doi:S0022-510X(07)00276-6 [pii]

Williams, J. H., Whiten, A., \& Singh, T. (2004). A systematic review of action imitation in autistic spectrum disorder. J Autism Dev Disord, 34(3), 285-299.

Wu, D., Jose, J. V., Nurnberger, J. I., \& Torres, E. B. (2018). A Biomarker Characterizing Neurodevelopment with applications in Autism. Sci Rep, 8(1), 614. doi:10.1038/s41598017-18902-w 
Figure 1: The task consisted of videos of the performance of a novel, meaningless gesture which lasted 4-8 seconds. Following a Go cue (fixation cross turning green), participants produced the gesture they had seen. They were then given non-specific feedback ("Correct," or, for example, "Notice how she moved her hand."). Thereafter, the white fixation cross appeared, and the same video replayed, for a total of 4-6 repetitions per gesture.

Figure 2: Learning curves by group for the first four repetitions of each gesture. The $y$-axis reflects number of gestures performed correctly (out of 8 possible), controlled for age, sex and motor coordination (quantified via PANESS). Whisker plots represent mean and 95\% confidence interval. Performance is statistically different between groups at repetition 3 but re-converges at repetition 4. Note that the upper limit of the $y$-axis extends beyond 8 , the total number of gestures scored; this is a consequence of adjustment for covariates.

Fig. 3. Causal Models Explicitly Tested in Analyses. (a) represents analyses of the pooled ASD+TD groups, whereas (b) represents a proposed causal model within the ASD group only. In both groups, we proposed that a learning mechanism, represented by the shape of the learning curve measured via principal component 1 (PC1) contributed directly toward overlearned praxis competence, as measured by performance on the pediatric modification of the Florida Apraxia Battery $(\mathrm{FAB})$, as represented by causal arrow $\delta$. We also proposed that the learning mechanism captured by PC1 influenced the FAB score indirectly, via ASD Dx (in the combined group) and/or ASD severity, measured by the ADOS in the ASD-only group; this relationship is represented by causal arrows $\alpha$ and $\gamma_{1}$. Motor coordination, as reflected by the Physical and Neurological Examination of Subtle Signs (PANESS), is known to be affected by ASD diagnosis 
and ASD severity; this relationship is represented by causal arrow $\beta$. Finally, motor coordination influences performance on the FAB (causal arrow $\gamma_{2}$ ).

Fig. 4. Loadings of the first principal component (PC1), representing a mean-shift of the performance at repetitions 1 through 4 . 


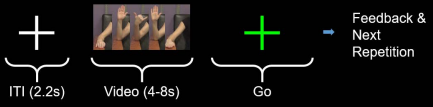




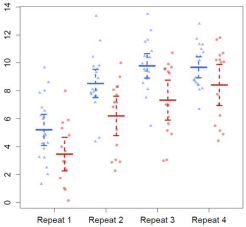

A TD $-\mathrm{HFA}$ 
(a) Motor Coordination (PANESS)

Learning Curve Shape (PC1)

\section{(b)}

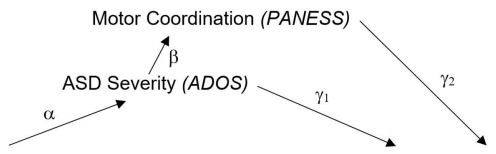

Learning Curve Shape (PC1)

- Overlearned Praxis Performance (FAB) $\delta$ 


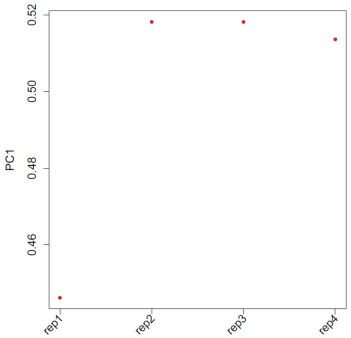

